\title{
EVALUACIÓN DE DOS NIVELES DE ENERGÍA EN EL COMPORTA- MIENTO PRODUCTIVO DE CUYES (Cavia porcellus) DE LA RAZA PERÚ
}

\author{
Effect of Two Energy Levels on the Productive Performance of Guinea \\ Pigs (Cavia porcellus) of the Peru Breed
}

\author{
Augusto Morales M. ${ }^{1}$, Fernando Carcelén C. ${ }^{1,2}$, Miguel Ara G. ${ }^{1}$, Teresa Arbaiza F. ${ }^{1}$, \\ Lilia Chauca F. ${ }^{3}$
}

\section{RESUMEN}

El objetivo del estudio fue determinar la respuesta en ganancia de peso, consumo de alimento, conversión alimenticia y rendimiento de carcasa en cuyes de raza Perú entre las 8 y 12 semanas de edad utilizando dos niveles energéticos, dietas isoproteicas (18\% PC), y uso de forraje. Los tratamientos 1 y 2 tuvieron 2.8 y 3.0 Mcal/kg ED con exclusión de forraje, y el tratamiento 3 (referencial) fue similar a T2 pero con suministro de forraje. El alimento y el agua se suministraron ad libitum. Se emplearon 72 cuyes machos, destetados, de $14 \pm 3$ días, distribuidos al azar en 24 pozas (8 pozas por tratamiento). No se encontró diferencia significativa en ganancias de peso vivo o en rendimiento de carcasa entre los tratamientos. Se registró un mayor consumo de materia seca total (5394 g) en T3 ( $<<0.05$ ). Los grupos T1 y T2 lograron una mejor conversión alimenticia a la semana 10 (3.18 y 3.32) que el grupo con forraje $(4.01)(\mathrm{p}<0.01)$.

Palabras clave: cuy, Cavia porcellus, energía, comportamiento productivo

\section{Abstract}

The aim of the study was to determine the response in terms of body weight gain, feed intake, feed conversion ratio, and carcass yield in guinea pigs of Peru breed between 8 and 12 weeks of age, using two isoproteic diets with different metabolizable energy levels (T1, 2.8; T2, 3.0 Mcal/kg DM) without forage and a third diet (T3) with 2.8 Mcal/kg plus forage. Feed and water were supplied ad libitum. Seventy two male weaned guinea pigs ( $14 \pm 3$ days of age) were randomly assigned to 24 rearing boxes (8 per treatment). None statistical difference was found between treatments on body weight gain or carcass yield. There was a better feed intake (total dry mater) in T3 $(\mathrm{p}<0.05)$. Groups T1 and T2 showed a better conversion ratio at week 10 (3.18 and 3.31) than T3 supplied with forage (4.01) $(\mathrm{p}<0.01)$.

Key words: guinea pig, Cavia porcellus, energy, productive performance

\footnotetext{
${ }^{1}$ Laboratorio de Bioquímica, Nutrición y Alimentación Animal, Facultad de Medicina Veterinaria, Universidad Nacional Mayor de San Marcos, Lima

${ }^{2}$ E-mail: nando2610@hotmail.com

${ }^{3}$ Instituto Nacional de Innovación Agraria (INIA), Lima
} 


\section{INTRODUCCIÓN}

El cuy es un pequeño mamífero doméstico, que destaca por su precocidad y prolificidad, que aunado a la excelente calidad de su carne, lo convierte en una fuente de proteínas capaz de competir con otras especies domésticas de interés productivo (Reymundo, 1984). El cuy es originario de la zona andina de Perú y Bolivia, donde fueron domesticados para ser usados principalmente en la alimentación humana (Aliaga, 1979). En la actualidad, la crianza de cuyes no solo constituye una buena alternativa alimenticia, sino también una importante fuente de ingresos, dada la gran demanda, como elemento principal en numerosos platos típicos que se expenden en fiestas patronales, fiestas regionales y ferias.

Los cuyes, como otras especies de interés zootécnico, requieren en su dieta diaria de diversos nutrientes en cantidades adecuadas, a fin de que alcancen su máxima tasa de crecimiento y eficiencia reproductiva. Cuantitativamente, el requerimiento energético es el más importante para los cuyes; sin embargo, las necesidades energéticas están influenciadas por la edad, actividad del animal, estado fisiológico, nivel y tipo de producción, y temperatura ambiental (Borja, 1979). La mayor parte de trabajos existentes sobre requerimientos nutricionales del cuy se han hecho considerando al cuy como animal de laboratorio y no como animal de producción. No obstante, se dispone de estudios donde no se encontraron diferencias en ganancia de peso, consumo y conversión alimenticia al comparar dietas con varios niveles de energía digestible (Torres et al., 2006), y en otros se pudo observar una respuesta sustancial en la respuesta animal al incremento energético (Castro y Chirinos, 1992). Asimismo, se ha encontrado una relación inversa entre el contenido de energía en la dieta y el consumo del alimento (Arroyo, 1986).

Se planteó el presente estudio con el fin de determinar la respuesta en crecimiento y acabado de cuyes de la raza Perú a dos dietas experimentales isoproteicas con diferente nivel energético. Se espera que una vez determinado el mejor nivel de energía para la mayor productividad, se pueda evaluar diversos insumos que, en combinación, proporcionen el nivel energético establecido al menor costo posible.

\section{Materiales y Métodos}

El estudio se llevó a cabo entre julio a setiembre de 2008 en el galpón de recría del Proyecto Cuyes (Programa de Animales Menores del Centro Experimental La Molina del INIA). Se empleó un galpón de 9 x 6 m, de cemento y ladrillo, con techo de fibrocemento, provisto de tragaluces, y con ventanas de malla metálica. Las pozas de recría fueron de cemento y ladrillo, de $0.8 \mathrm{x}$ $0.4 \times 0.45 \mathrm{~m}$, a $30 \mathrm{~cm}$ por encima del suelo, $\mathrm{y}$ equipadas con un comedero para concentrado de $500 \mathrm{~g}$ de capacidad y un bebedero de $750 \mathrm{ml}$.

Las dietas experimentales fueron elaboradas en la Planta de Alimentos de la Universidad Nacional Agraria La Molina. Los análisis químicos se efectuaron en el Centro Experimental La Molina.

Se trabajó con 72 cuyes Perú, machos, recién destetados, de 11 a 17 días de edad, provenientes de camadas de 3 a 4 crías. Los animales se distribuyeron aleatoriamente en 24 pozas de recría. Los cuyes y el alimento se pesaron con una balanza electrónica de $10 \mathrm{~kg}$ con $2 \mathrm{~g}$ de sensibilidad. El experimento duró10 semanas.

Las pozas fueron distribuidas aleatoriamente en tres tratamientos, que consistieron en tres dietas isoproteicas con dos niveles de energía y de forraje: T1 con 2.8 y T2 con 3.0 Mcal/kg de energía digestible (ED), ambos con exclusión de forraje; y T3, con $2.8 \mathrm{Mcal} / \mathrm{kg}$ y con suministro de forraje (tratamiento referencial). En el Cuadro 1 se presenta la composición de las dos dietas ex- 
perimentales y en el Cuadro 2 su valor nutricional. Las dietas se formularon con el programa Mixit 2 para un nivel de 18\% de proteína, $10 \%$ de fibra cruda, y los niveles mínimos requeridos de aminoácidos y vitamina C (Cuadro 3). Los valores proximales promedio del forraje (maíz chala) fueron (en base fresca) de $76.5 \%$ de humedad, $2.46 \%$ de proteína, $0.36 \%$ de extracto etéreo, $5.9 \%$ de fibra cruda, $13.23 \%$ de extracto libre de $\mathrm{N}$ y $1.57 \%$ de ceniza.

Las variables de respuesta evaluadas fueron ganancia de peso vivo, conversión alimenticia y rendimiento de carcasa. La ganancia de peso se calculó en base a la diferencia entre el peso final y el peso inicial, obtenidos al inicio del experimento y semanalmente desde la semana 8 hasta la semana 12 de edad. El consumo de alimento fue estimado por tratamiento y por poza a partir de la diferencia entre el alimento ofrecido y el residuo, tomando en cuenta el desperdicio. La evaluación del consumo se hizo a las 8, 10 y 12 semanas de edad. La conversión alimenticia semanal se calculó en base al consumo semanal de alimento en base seca (g) y la ganancia semanal de peso (g), en tanto que la conversión alimenticia acumulada se calculó en base al consumo acumulado de alimento en base seca (g) y la ganancia acumulada de peso (g).

El rendimiento de carcasa a las 8, 10 y 12 semanas se estableció a través de dos animales por tratamiento, seleccionados al azar, que se beneficiaron previo ayuno de 24 horas. La carcasa incluyó piel, cabeza, patas y órganos internos (corazón, pulmón, hígado, bazo y riñón).

Se utilizó un diseño completamente al azar con tres tratamientos y ocho repeticiones, considerándose a cada poza con tres cuyes como una unidad experimental. La comparación entre medias de tratamientos se hizo con la prueba de rango múltiple de Duncan (Calzada, 1982). Todas las pruebas estadísticas fueron hechas con un nivel de significación de 0.05 .

\section{Resultados y Discusión}

La ganancia promedio de peso hasta las 8 semanas, y entre las semanas 9, 10 y 12 de edad no fue estadísticamente diferente entre tratamientos. La ganancia acumulada de peso a las 8 semanas fue de 629, 599 y 623 para los tratamientos T1, T2 y T3, respectivamente, sin diferencias estadísticas entre grupos. Asimismo, el peso individual de los cuyes a las 12 semanas fue de 1203, 1202 y 1213 g para los tratamientos T1, T2 y T3; sin embargo, los animales alcanzaron pesos comerciales (>850 g) a partir de la octava semana de evaluación. Las ganancias de peso para el tratamiento con 3.0 Mcal ED/kg PV fue ligeramente superior al reportado por Torres et al. (2006), diferencia debida a que en ese estudio hubo inclusión de forraje.

Los consumos totales (tal como ofrecido) de alimento balanceado, forraje, y el consumo total de materia seca de los tratamientos T1, T2 y T3 se muestran en el Cuadro 4. El consumo diario y total de alimento balanceado fue significativamente mayor en los tratamientos sin forraje (T1 y T2). Las diferencias en consumo del alimento balanceado entre T2 y T3 fueron consistentes a favor de T2, aunque sin significancia estadística, lo que sugeriría que los animales tienden a un mayor consumo a medida que se reduce el nivel de energía en la dieta (Samamé, 1983), posiblemente debido a una compensación por el menor aporte nutricional de la dieta (Inga, 2008).

Los grupos tratados con alimento balanceado y exclusión de forraje (T1 y T2) lograron una mejor conversión alimenticia a la semana 10 (3.18 y 3.32) que el grupo con forraje (4.01) $(\mathrm{p}<0.01$, Cuadro 5). La conversión de 2.75 en la semana 9 para T1 fue mejor que aquella de 3.3 reportada por Torres et al. (2006) para el mismo nivel energético, y más cercana a la de 2.86 obtenida por Inga (2008); diferencias explicables si se toma en consideración la composición del alimento suministrado. 
Cuadro 1. Composición porcentual de las dietas experimentales

\begin{tabular}{lcc}
\hline Ingredientes & 3.0 ED 10\% FC & 2.8 ED 10\% FC \\
\hline Maíz amarillo & 28.310 & 29.937 \\
Torta de soya & 19.202 & 15.825 \\
Subproducto de trigo & 18.836 & 21.950 \\
Cascarilla de arroz & 6.000 & 6.000 \\
Heno de alfalfa & 16.043 & 13.02 \\
Pasta de algodón & 5.867 & 10.00 \\
Aceite vegetal & 2.978 & 0.564 \\
Fosfato dicálcico & 1.682 & 1.424 \\
Sal & 0.409 & 0.407 \\
Carbonato de calcio & 0.147 & 0.421 \\
Cloruro de colina & 0.150 & 0.150 \\
Premix & 0.120 & 0.120 \\
Mold Zap & 0.100 & 0.100 \\
Rovimix & 0.080 & 0.080 \\
L-lisina & 0.018 & 0.000 \\
DL-Metionina & 0.058 & 0.002 \\
\hline TOTAL & 100.0 & 100.0 \\
\hline Costo $(\$ / k g)$ & 0.35 & 0.31 \\
\hline
\end{tabular}

Cuadro 2. Valor nutritivo estimado de las dietas experimentales

\begin{tabular}{lcc}
\hline Nutrientes & 3.0 ED 10\% FC & 2.8 ED 10\% FC \\
\hline ED (Mcal/kg) & 2.95 & 2.80 \\
Proteína (\%) & 18.00 & 18.00 \\
Fibra (\%) & 10.00 & 10.00 \\
Grasa (\%) & 5.00 & 2.794 \\
Lisina (\%) & 0.930 & 0.887 \\
Metionina (\%) & 0.342 & 0.290 \\
Metionina + cistina (\%) & 0.660 & 0.632 \\
Arginina (\%) & 1.320 & 1.398 \\
Triptofano (\%) & 0.286 & 0.284 \\
Treonina (\%) & 0.723 & 0.717 \\
Calcio (\%) & 0.80 & 0.80 \\
P total (\%) & 0.80 & 0.80 \\
Sodio (\%) & 0.20 & 0.20 \\
Vitamina C (mg/kg) & 280.0 & 280.0 \\
\hline
\end{tabular}


Cuadro 3. Análisis proximal porcentual de las dietas experimentales (base fresca)

\begin{tabular}{lccc}
\hline & \multicolumn{3}{c}{ Tratamiento } \\
\cline { 2 - 4 } & 1 & 2 & Control \\
\hline ED (Mcal/kg) & 3.0 & 2.8 & 2.8 \\
Fibra cruda (\%) & 10 & 10 & 10 \\
Nutrientes (\%) & & & \\
Humedad & 12.13 & 12.78 & 12.78 \\
Materia seca & 87.87 & 87.22 & 87.22 \\
Proteína & 18.62 & 17.83 & 17.83 \\
Fibra cruda & 10.36 & 8.84 & 8.84 \\
Extracto etéreo & 5.38 & 3.35 & 3.35 \\
Ceniza & 6.99 & 6.57 & 6.57 \\
E.L.N. & 46.53 & 50.63 & 50.63 \\
\hline
\end{tabular}

Cuadro 4. Consumo promedio ( 8 repeticiones con tres cuyes por poza) alimentos (en gramos) tal como ofrecido y en materia seca en cuyes Perú sometidos a dietas isoproteicas con dos niveles de energía digestible y uso de forraje

\begin{tabular}{lcccc}
\hline $\begin{array}{l}\text { Consumo } \\
(\mathrm{g})\end{array}$ & $\begin{array}{c}\text { Edad } \\
\text { (semanas) }\end{array}$ & $\begin{array}{c}\mathrm{T} 1 \\
(3.0 \mathrm{ED})\end{array}$ & $\begin{array}{c}\mathrm{T} 2 \\
(2.8 \mathrm{ED})\end{array}$ & $\begin{array}{c}\mathrm{T} 3 \\
(2.8 \mathrm{ED}+ \\
\text { forraje })\end{array}$ \\
\hline \multirow{3}{*}{ Alimento balanceado } & 8 & $1858^{\mathrm{a}}$ & $1972^{\mathrm{a}}$ & $1641^{\mathrm{b}}$ \\
& 10 & $2798^{\mathrm{b}}$ & $2900^{\mathrm{a}}$ & $2439^{\mathrm{c}}$ \\
& 12 & $3892^{\mathrm{a}}$ & $3967^{\mathrm{a}}$ & $3327^{\mathrm{b}}$ \\
Forraje & 8 & 0 & 0 & 2301 \\
& 10 & 0 & 0 & 3722 \\
& 12 & 0 & 0 & 5404 \\
Materia seca (total) & 8 & $1636^{\mathrm{b}}$ & $1732^{\mathrm{b}}$ & $2648^{\mathrm{a}}$ \\
& 10 & $2464^{\mathrm{b}}$ & $2547^{\mathrm{b}}$ & $4152^{\mathrm{a}}$ \\
& 12 & $3427^{\mathrm{b}}$ & $3484^{\mathrm{b}}$ & $5934^{\mathrm{a}}$ \\
\hline
\end{tabular}

a,b,c Consumos con superíndices con letras diferentes, dentro de cada tipo de consumo y edad, son estadísticamente diferentes $(p<0.05)$

El rendimiento de carcasa varió entre 71 y $72.8 \%$ a las 8 semanas de edad, y entre 71.9 a $75.6 \%$ en la semana 12 , sin haber diferencias significativas entre tratamientos. Sin embargo, los resultados del presente estudio se basan en una muestra muy pe- queña de observaciones por tratamiento, por lo que los resultados deben ser tomados con cautela. Los rendimientos de carcasa fueron similares a otros estudios (Torres et al., 2006; Inga, 2008), aunque superiores a los reportados por Chauca (1997). 
Cuadro 5. Conversión alimenticia promedio $(n=8)$ de cuyes Perú sometidos a diferentes niveles energéticos al final de cuatro periodos de evaluación

\begin{tabular}{cccc}
\hline Edad (semanas) & T1 (3.0 ED) & T2 (2.8 ED) & $\begin{array}{c}\text { T3 }(2.8 \text { ED + } \\
\text { forraje })\end{array}$ \\
\hline 8 & $2.63^{\mathrm{a}}$ & $2.95^{\mathrm{a}}$ & $3.23^{\mathrm{a}}$ \\
9 & $2.75^{\mathrm{a}}$ & $3.06^{\mathrm{a}}$ & $3.55^{\mathrm{a}}$ \\
10 & $3.18^{\mathrm{a}}$ & $3.32^{\mathrm{a}}$ & $4.01^{\mathrm{b}}$ \\
12 & $3.74^{\mathrm{a}}$ & $3.81^{\mathrm{a}}$ & $4.40^{\mathrm{a}}$ \\
\hline
\end{tabular}

a,b Valores con superíndices diferentes dentro de semanas de edad tienen significancia estadística $(\mathrm{p}<0.01)$

\section{Conclusiones}

- El peso final y la ganancia de peso tuvieron un comportamiento similar como respuesta a las dietas experimentales sin uso de forraje.

- El mayor nivel energético nutricional de la dieta ocasionó un menor consumo de alimento y por lo mismo una mejor conversión alimenticia.

\section{LiTERATURA Citada}

1. Aliaga R. 1979. Producción de cuyes. Huancayo: Universidad Nacional del Centro. 58 p.

2. Arroyo O. 1986. Avances de investigación sobre cuyes en el Perú. Series de Informes Técnicos 7. Lima: Proyecto PISA, INIPA, CIID, ACDI. 331 p.

3. Borja A. 1979. Nutrición. En: Producción de cuyes. Huancayo: Universidad Nacional del Centro. p 141-181.

4. Calzada J. 1982. Métodos estadísticos para la investigación. Lima: Ed Jurídica. $644 \mathrm{p}$.

5. Castro J, Chirinos D. 1992. Uso de tres niveles energéticos en suplementos para cuyes destetados y el efecto de la adición de la tiroproteína. En: XV Re- unión de la Asociación Peruana de Producción Animal. Pucallpa: APPA.

6. Chauca L. 1997. Producción de cuyes (Cavia porcellus). Organización de las Naciones Unidas para la Agricultura y Alimentación. Roma: FAO. 120 p. [Internet], [6 diciembre 2008]. Disponible en http://www.fao.org/docrep/ w6562s/w6562s00.HTM

7. Inga R. 2008. Evaluación de dos niveles de energía digestible y dos niveles de fibra cruda en dietas de crecimiento, con exclusión de forraje para cuyes raza Perú (Cavia porcellus). Tesis de Ingeniero Zootecnista. Lima: Univ Nacional Agraria La Molina. 74 p.

8. Reymundo B. 1984. Concentrado ofrecido al inicio y/o acabado y su efecto en la velocidad de crecimiento en cuyes. Tesis de Ingeniero Zootecnista. Huancayo: Universidad Nacional del Centro. $88 \mathrm{p}$.

9. Samamé J. 1983. Niveles de energía en cuyes en reproducción y en crecimiento. Tesis de Ingeniero Zootecnista. Lima: Universidad Nacional Agraria La Molina. $96 \mathrm{p}$.

10. Torres A, Chauca L, Vergara V. 2006. Evaluación de dos niveles de energía y proteína en dietas de crecimiento y engorde de cuyes machos. En: XXIX Reunión de la Asociación Peruana de Producción Animal. Huancayo: APPA. 